\title{
EXETER
}
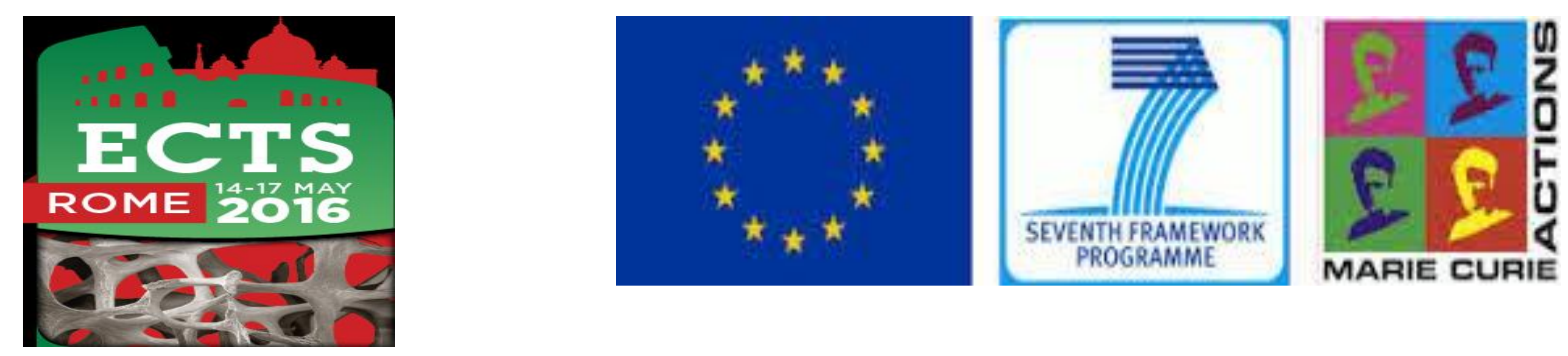

\section{UK popular sports and hip differences on bone outcomes in adolescent male athletes: The PRO-BONE study.}

Dimitris Vlachopoulos ${ }^{1}$, Alan R. Barker ${ }^{1}$, Craig A. Williams ${ }^{1}$, Sigurbjörn A. Arngrímsson ${ }^{3}$, Karen M. Knapp ${ }^{5}$, Brad S. Metcalf ${ }^{1,5}$, Ioannis G. Fatouros ${ }^{4}$, Luis A. Moreno ${ }^{2}$, Luis Gracia-Marco ${ }^{1,2}$

${ }^{1}$ Children's Health and Exercise Research Centre. Sport and Health Sciences, University of Exeter, United Kingdom.

${ }^{2}$ Growth, Exercise, Nutrition and Development Research Group, University of Zaragoza, Spain.

${ }^{3}$ Center for Sport and Health Sciences, University of Iceland, Iceland.

${ }^{4}$ Physical Education and Sport Sciences, University of Thessaly, Greece.

${ }^{5}$ University of Exeter Medical School, United Kingdom.

\section{Introduction}

Sports specific training may have different impact on bone outcomes, and adolescence is a key period to optimize bone health. The aim was to examine hip differences on bone outcomes between osteogenic (football) and nonosteogenic (swimming and cycling) sports in adolescent males.

\section{Methods}

One hundred twenty one males $(13.1 \pm 0.1$ years $)$ volunteered to partake in this study that received ethics approval from the UK National Research Ethics Service. Participants included: 41 swimmers, 37 footballers, 29 cyclists and 14 age matched controls. Participants in the sport groups had engaged in sport-specific training for $\geq 3$ hours/week for the last 3 years. Bone mineral density (BMD) and bone mineral content (BMC) were determined at the hip sites using dual energy X-ray absorptiometry. Hip structural analysis (HSA) software was used to obtain bone geometry parameters at the femoral neck. Results were adjusted for age, height, lean mass, calcium intake and physical activity.

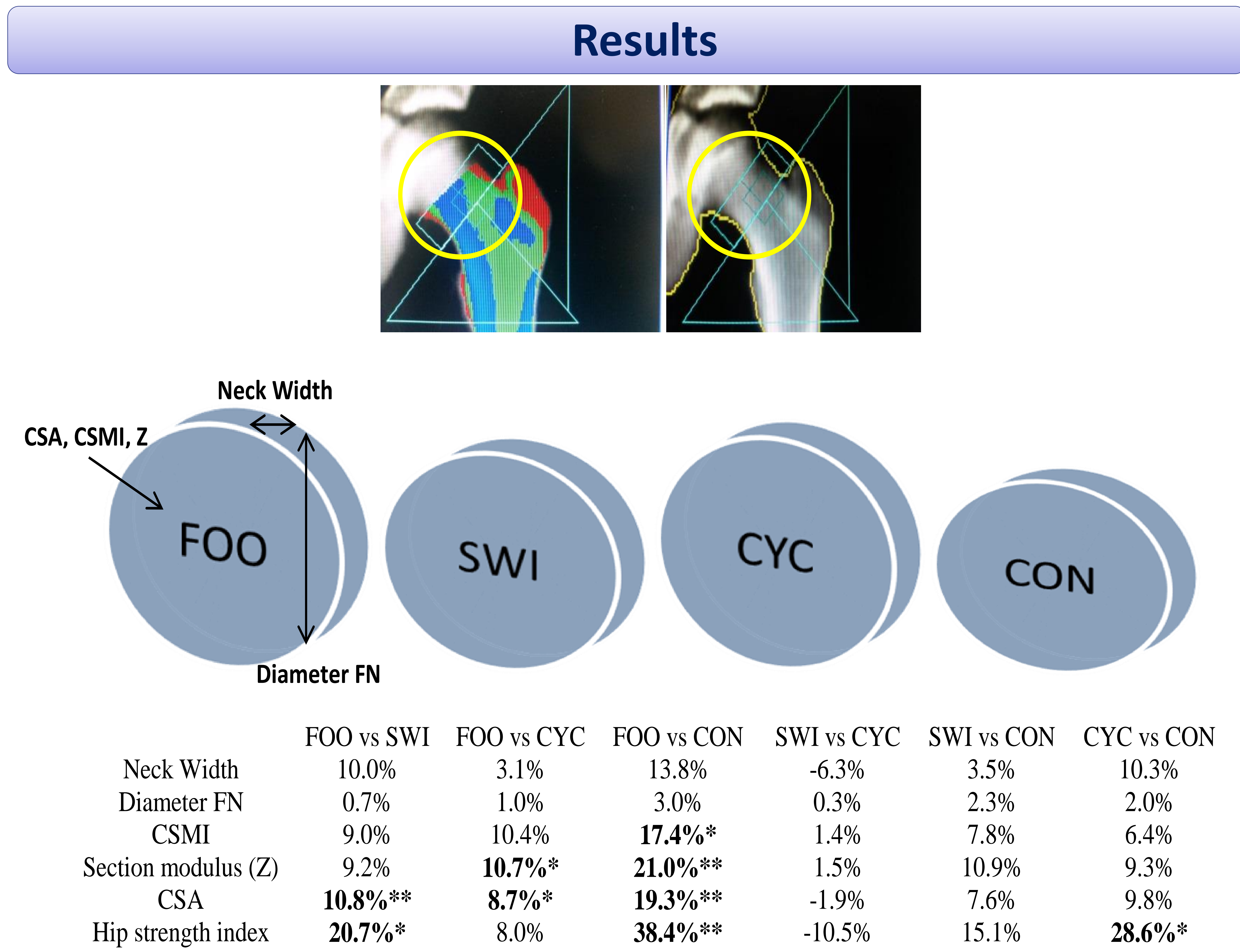

Figure 1: Differences in adjusted geometrical parameters of the narrow neck site between groups.

Footballers had significantly higher BMD and BMC at total hip (10$23 \%)$, trochanter (13-20\%), shaft (10-20\%) and Ward's triangle (13-28\%) sites compared to all the other groups. Footballers' BMD was also higher at femoral neck compared to the control group (19\%). Footballers showed (Figure 1) higher cross-sectional area compared to all the groups (9-19\%), cross-sectional moment of inertia compared to controls (17\%) and section modulus compared to cyclists (11\%) and controls (21\%). Hip strength index (HSI) was significantly higher in cyclists compared to controls $(29 \%)$ and in footballers compared to swimmers $(21 \%)$ and controls $(38 \%)$. All results above were significant at $p<0.05$.

\section{Conclusions}

Adolescent male footballers have an enhanced BMD, BMC and bone geometry parameters compared to the other groups at a clinically relevant site such as the hip. In addition, cyclists also had significantly higher HSI than controls. 